\title{
Un modelo educativo para la clasificación de factores que afectan la eficiencia de la reurbanización del entorno y la reubicación de empresas industriales
}

\section{An educational model for the classification of factors that affect the efficiency of the redevelopment of the environment and the relocation of industrial companies}

\author{
Azariy Lapidus $^{1 \mathrm{a}}$ \\ Moscow State University of Civil Engineering, Moscow, Russia ${ }^{1}$ \\ ORCID ID: https://orcid.org/0000-0001-7846-5770¹
}

Recibido: 14 de setiembre de 2020

Aceptado: 15 de enero de 2021

\begin{abstract}
Resumen
El propósito de este estudio fue determinar la jerarquía de factores fundamentales que determinan la posibilidad de reubicación de empresas industriales para racionalizar la infraestructura urbana. Mediante el análisis de la literatura científica y el método de evaluación de expertos, se ha elaborado una lista de factores prioritarios que inciden en la posibilidad de reurbanización del entorno urbano y la reubicación de una instalación industrial. El uso de la evaluación experta y la escala Likert estableció relaciones causales entre factores, se ha desarrollado un modelo de jerarquía de factores mediante el método gráfico, a partir de cuyos resultados se ha establecido una estructura de factores de 5 niveles que inciden en la posibilidad de remodelación del entorno urbano y reubicación de una instalación industrial. Los resultados obtenidos pueden ser útiles para estructuras empresariales, así como para organismos gubernamentales. Parece que una consideración más completa de los siguientes factores no protegerá por completo los proyectos para el redesarrollo de zonas industriales en las ciudades rusas de elegir los conceptos incorrectos, no de la recuperación, el bajo interés de los turistas, sino que reducirá la probabilidad de que no decisiones óptimas.
\end{abstract}

Palabras clave: redespliegue, empresas industriales, infraestructura urbana, remodelación, reubicación, ciudad, racionalización.

\begin{abstract}
The purpose of this study was to determine the hierarchy of fundamental factors determining the possibility of relocation of industrial enterprises in order to rationalize urban infrastructure. By analyzing scientific literature and the method of expert assessments, a list of priority factors has been formed that affect the possibility of redeveloping the urban environment and the relocation of an industrial facility. The use of expert assessment and the Likert scale established
\end{abstract}


causal relationships between factors. A model of the hierarchy of factors has been developed using the graph method, based on the results of which a 5-level structure of factors has been established that affect the possibility of redeveloping the urban environment and relocation of an industrial facility. The obtained results can be useful for business structures, as well as government bodies. It seems that a fuller consideration of the following factors will not so much completely protect projects for the redevelopment of industrial zones in Russian cities from choosing the wrong concepts, not payback, low interest from tourists, but rather reduce the likelihood of making completely non-optimal decisions.

Keywords: redeployment, industrial enterprises, urban infrastructure, redevelopment, relocation, city, rationalization.

\section{Introduction}

Most cities in the Russian Federation found themselves at the junction of two industrial revolutions - one is still driving outdated factories and factories from megacities, and the second is already attracting new high-tech industries to the interior of the city by the availability of a high-quality workforce and centers of competence (Auzan, 2017). The layering of these trends in the development of urban infrastructure in modern conditions is the main threat to the effectiveness of the implementation of the industrial policy of a modern metropolis and urban infrastructure (Yanitsky, 2018). If we consider, for example, the European experience, then the development of market relations led to an increase in the value of land plots in the center of cities, which, in turn, displaced industrial enterprises to the outskirts and outside of megacities. In Russia, with the development of market relations, the industrial sector retreated under the pressure of the growth in the value of land plots, which was a natural regulator of the city's development. In the conditions of the Soviet period, the very concept of "land value" did not exist a priori (Aleskerov, 2019). The so-called industrial zones occupy thousands of hectares. In most of them, production was stopped many years ago (Aleskerov, 2019). Chaotic warehouses and landfills appeared on unused areas. They spoil the appearance of the capital, use expensive land ineffectively, and worsen the ecological situation.

Incompatibility of industrial companies built over time in the Union of Soviet Socialist Republics (USSR) with today's requirements, significant technological progress, economic reform, and transition to the market principles for evaluating efficiency imply the fact that their target and functional purposes must be changed (Gokhberg, et al., 2020; Kosyakov \& Guskov, 2019). However, technical conditions of many industrial buildings in the Russian Federation allow them to be in operation for more than one decade. Most of these buildings are in settlements. Therefore, development of cities led to a significant increase in their areas, as a result of which industrial companies once built on the outskirts were within or even in the 
central parts of cities (Power, 2018; Lapidus, \& Topchiy, 2019; Abid, Saeed, \& Al-Beyaty, 2019).

Redeployment of production companies means that construction costs and provision of new facilities within a city. Despite many industrial buildings that have lost their effectiveness and relevance, as along with significant interest from investors in these facilities, the field of construction redeployment remains poorly studied. Redeployment is the transfer of production coefficients from one area of use to another. For example, an industrial building redeployment is a change in its functional purposes to change the operational qualities, increase the profitability of the facility, and consolidate this procedure legally in the relevant government institutions (Magnusson, Berggren, 2018; Mäkitie, 2019).

When a construction company is engaged in the construction or redevelopment of complex industrial facilities, then it separately analyzes all the factors affecting different parts of the facilities (Lai et al., 2020; Lonati, Saponaro, Sezenna, 2019; Wang, 2019). This ensures the smooth and efficient operation of the industrial facility construction company. The process of sequential erection of construction objects is necessary in order to begin construction as soon as possible and determine the priority objects. The most optimal option for the construction of buildings is possible only thanks to clear planning and taking into account all building factors (Lonati, Saponaro, Sezenna, 2019; Wang, 2019; Topchiy et al., 2019). Therefore, for any industrial facility it is necessary to carry out preliminary calculations and analysis. This will ensure an even distribution of construction work and the stability of their implementation.

However, to achieve and strengthen long-term competitiveness, companies are forced to adjust their activities with an emphasis on the changing demands of the period. Since the world is constantly changing, it is very important to respond to these changes expediently and quickly. Consequently, it must be performed only in the presence of the clearly defined goals, redeployment concept, and an understanding of each stage and the methods to be observed. This is a relevant study because the model of the work performed during redeployment allows this process to go as smoothly and efficiently as possible allowing the company to adapt to new market conditions. Therefore, it is necessary to define and ascertain the entire system of factors influencing the redevelopment of industrial buildings for the rationalization of urban infrastructure. Within the framework of this study, the determining factors of the relocation of industrial enterprises were identified in order to rationalize the urban infrastructure, and the priority of their influence was established, which can contribute to the formation of an effective strategy for the redevelopment of industrial territories with the rationalization of urban infrastructure. 
Likewise, this work has a pedagogical approach, as it presents the theme of urbanization of the environment as a working model in architecture and civil engineering so that future students and experts in urban models can have a work that guides in this regard. That is the educational function of this study.

\section{Methods and materials}

The methodological approach to assessing the feasibility of re-profiling the urban environment is based on prioritizing the organizational and technical factors that affect the possibility of re-profiling the urban environment by building a hierarchical model. To construct a hierarchy model, the method of graphs was used, which makes it possible to structure factors by levels based on the mutual influence and interdependencies between them. In order to implement the method with a set of factors $(Z)$, two subsets are distinguished: $S\left(z_{i}\right)$ is the reachability subset and $P(z i)$ is the subset of predecessor vertices. A $z j$ vertex is called reachable if there is a path in the graph that leads from the $z_{i}$ vertex to the $z_{j}$ vertex $\left(b_{i j}=1\right)$ (Kühn et al., 2017):

$$
b_{i j}=\left\{\begin{array}{c}
1, \text { if theivertexleadstothejvertexbasedontheorientedgraph; } \\
0, \text { iftheivertexdoesnotleadtothejvertexbasedontheorientedgraph }
\end{array}\right.
$$

where $b_{i j}$ is the assessment of reachability of the $j$ th vertex through the $i$ th vertex.

The $z_{i}$ vertex is the predecessor of the $z_{j}$ vertex if the second one reaches the apex of the first one. Those vertices for which condition (2) is satisfied form the first (lowest) level of the hierarchy. At the 2nd iteration, the objects that formed the 1st level of the hierarchy are excluded. The analysis continues until all factors have been graded (Kühn et al., 2017).

$$
P\left(z_{i}\right)=S\left(z_{i}\right) \cap P\left(z_{i}\right)
$$

For the other hand, because of the purpose of the study, an expert group was formed, consisting of 50 experts - specialists from the Department of Urban Development Policy of the city of Moscow, the Ministry of Industry and Trade of the Russian Federation, and the Federal Agency for Tourism.

The proposed list of factors affecting the possibility of repurposing the urban environment and relocating an industrial facility is based on (Magnusson, Berggren, 2018; Mäkitie, 2019; Lai, et al., 2020; Lonati, Saponaro, Sezenna, 2019; Wang, 2019) and includes: 
F1 - the factor of the availability of natural resources; F2 - the factor of the availability of labor resources and their cost; F3 - the factor of access to technology; F4 - the factor in the development of science in the region; F5 - the climatic factor; F6 - the factor of investment attractiveness of the region; F7 - the factor of the availability of sales markets; F8 - the factor of competition; F9 - the factor in the development of production, engineering, transport, economic infrastructure; F10 - the factor in the development of social, tourist infrastructure; F11 - the factor in the development of information and communication technologies; F12 the factor of ecological compatibility of the region; F13 - the factor of historical and cultural value of the region; F14 - the legislative factor; F15 - the neighborhood factor; F16 - the topological factor; F17 - the personal factor.

The degree of representativeness of the factors F1-F17 was estimated by experts at 89.2\% (223 out of 250 maximum possible points for the entire expert group), which indicates the representativeness of the proposed list of factors that affect the possibility of repurposing the urban environment and relocating an industrial facility.

\section{Results}

The scores set according to the results of the survey, which characterize the possibility of the influence of one factor on another, are given in Table 1. The table shows the average scores for the expert group. The given points characterize the degree of direct influence (on a 5-point Likert scale) of the ith factor on the jth.

Table 1.

Expert assessments of the interdependencies between factors affecting the possibility of repurposing the urban environment and relocation of an industrial facility

\begin{tabular}{|c|c|c|c|c|c|c|c|c|c|c|c|c|c|c|c|c|c|}
\hline ith & \multicolumn{17}{|c|}{ jth factor } \\
\hline factor & F1 & F2 & F3 & F4 & F5 & F6 & F7 & F8 & F9 & F10 & F11 & F12 & F13 & F14 & F15 & F16 & F17 \\
\hline F1 & - & 1 & 1 & 1 & 1.2 & 4.3 & 2.7 & 2.9 & 4.2 & 3.8 & 1 & 2.9 & 1 & 1 & 1 & 1 & 1.1 \\
\hline F2 & 1 & - & 3.5 & 4.1 & 1 & 4.4 & 3.6 & 3.8 & 4.4 & 4 & 3.8 & 1 & 1 & 1 & 1 & 1 & 2.4 \\
\hline F3 & 1.2 & 1 & - & 4.3 & 1 & 4.2 & 3.5 & 3.8 & 4.6 & 4.2 & 4.4 & 1.4 & 1.2 & 1.3 & 1 & 1 & 1 \\
\hline $\mathrm{F} 4$ & 1 & 1.7 & 4.8 & - & 1 & 5 & 2.1 & 4 & 5 & 4.7 & 5 & 1 & 1 & 1 & 1 & 1 & 1 \\
\hline F5 & 5 & 2.7 & 1 & 1 & - & 3.6 & 1.7 & 3.7 & 4 & 4 & 2.7 & 4.1 & 3.7 & 3.7 & 1 & 4.4 & 1 \\
\hline F6 & 1 & 3.6 & 3.7 & 4 & 1 & - & 1 & 4 & 4 & 4 & 3.6 & 1 & 1 & 1 & 1 & 1 & 3.6 \\
\hline F7 & 1 & 3.9 & 3.6 & 3.5 & 1 & 4.1 & - & 5 & 5 & 4.4 & 4.3 & 1 & 1 & 1 & 1 & 1 & 1 \\
\hline $\mathrm{F} 8$ & 1 & 3.7 & 3.7 & 4 & 1 & 3.2 & 3.3 & - & 3.9 & 3.8 & 3.4 & 1 & 1 & 1 & 1 & 1 & 1 \\
\hline F9 & 1 & 3.6 & 4 & 4 & 1 & 5 & 3.9 & 5 & - & 4.1 & 3.6 & 1 & 1 & 1 & 1 & 1 & 1 \\
\hline F10 & 1 & 4 & 4 & 4 & 1 & 4.8 & 3.9 & 4.1 & 3.9 & - & 3.9 & 1 & 1 & 1 & 1 & 1 & 1 \\
\hline F11 & 1 & 3.6 & 3.9 & 3.8 & 1 & 4.3 & 3.2 & 3.8 & 4.6 & 3.7 & - & 1 & 1 & 1 & 1 & 1 & 1 \\
\hline F12 & 4.6 & 2.4 & 3.8 & 2.9 & 1 & 3.9 & 2.5 & 2.3 & 4 & 4.6 & 3.6 & - & 4.8 & 5 & 1 & 1 & 3.9 \\
\hline F13 & 4.6 & 2.6 & 3.6 & 3.7 & 1 & 2.8 & 2.2 & 2.6 & 3.8 & 4.2 & 2.5 & 5 & - & 5 & 1 & 1 & 3.9 \\
\hline F14 & 3.6 & 2.8 & 2.7 & 2.6 & 1 & 5 & 3.8 & 4.1 & 4.6 & 4.8 & 3.6 & 5 & 4 & - & 1 & 1 & 2.4 \\
\hline F15 & 2.3 & 3.6 & 3.2 & 3.4 & 1 & 3.9 & 4.1 & 4.1 & 4.2 & 4.2 & 3.9 & 1.2 & 2.1 & 1.1 & - & 1 & 3.6 \\
\hline F16 & 4.3 & 3.6 & 2.9 & 3 & 5 & 3.8 & 3.6 & 3.7 & 4.1 & 3.9 & 3 & 4.1 & 4.1 & 3.9 & 1 & - & 1 \\
\hline F17 & 1 & 3.5 & 1.2 & 1.2 & 1 & 1.3 & 1.7 & 2 & 1.1 & 1.1 & 1 & 1 & 1 & 1 & 1 & 1 & - \\
\hline
\end{tabular}


The obtained results are interpreted as follows: estimates in the range of 1.00-1.80 indicate the absence of influence of the ith factor on the jth, 1.81-2.60 - about an insignificant effect, 2.61-3.40 - about an average force of influence, 3.41-4.20 - high, 4.21- 5.00 is very high. These levels are obtained by dividing the range of possible estimates $[1 ; 5]$ at 5 equal intervals. For the implementation of the next stage of the study - determination of the levels of the hierarchy of factors, interconnections were used, the strength of which was assessed at a high and very high level ( $\geq 3.41$ ). The results of determining the levels of the hierarchy of factors using formulas (1) - (2) are shown in Tables 2-6.

Table 2.

Levels of the hierarchy of factors that affect the possibility of repurposing the urban environment and relocation of an industrial facility (1st iteration)

\begin{tabular}{|c|c|c|c|c|}
\hline $\begin{array}{c}\text { Fact } \\
\text { or }\end{array}$ & $\mathrm{S}(\mathrm{zi})$ & $\mathrm{P}(\mathrm{zi})$ & $\mathrm{S}(\mathrm{zi}) \cap \mathrm{P}(\mathrm{zi})$ & Level \\
\hline F1 & $\begin{array}{c}\text { F1, F2, F3, F4, F6, F7, F8, F9, F10, F11, } \\
\text { F17 }\end{array}$ & F1, F5, F12, F13, F14, F16 & $\mathrm{F} 1$ & - \\
\hline $\mathrm{F} 2$ & F2, F3, F4, F6, F7, F8, F9, F10, F11, F17 & $\begin{array}{l}\text { F1, F2, F3, F4, F5, F6, F7, F8, F9, } \\
\text { F10, F11, F12, F13, F14, F15, F16, } \\
\text { F17 }\end{array}$ & $\begin{array}{l}\text { F2, F3, F4, F6, F7, } \\
\text { F8, F9, F10, F11, } \\
\text { F17 }\end{array}$ & - \\
\hline F3 & F2, F3, F4, F6, F7, F8, F9, F10, F11, F17 & $\begin{array}{l}\text { F1, F2, F3, F4, F5, F6, F7, F8, F9, } \\
\text { F10, F11, F12, F13, F14, F15, F16, } \\
\text { F17 }\end{array}$ & $\begin{array}{l}\text { F2, F3, F4, F6, F7, } \\
\text { F8, F9, F10, F11, } \\
\text { F17 }\end{array}$ & - \\
\hline F4 & F2, F3, F4, F6, F7, F8, F9, F10, F11, F17 & $\begin{array}{l}\text { F1, F2, F3, F4, F5, F6, F7, F8, F9, } \\
\text { F10, F11, F12, F13, F14, F15, F16, } \\
\text { F17 }\end{array}$ & $\begin{array}{c}\text { F2, F3, F4, F6, F7, } \\
\text { F8, F9, F10, F11, } \\
\text { F17 }\end{array}$ & - \\
\hline F5 & $\begin{array}{c}\text { F1, F2, F3, F4, F5, F6, F7, F8, F9, F10, } \\
\text { F11, F12, F13, F14, F16, F17 }\end{array}$ & 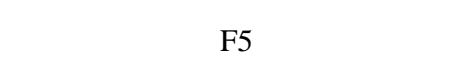 & F5 & 1 \\
\hline F6 & F2, F3, F4, F6, F7, F8, F9, F10, F11, F17 & $\begin{array}{c}\text { F1, F2, F3, F4, F5, F6, F7, F8, F9, } \\
\text { F10, F11, F12, F13, F14, F15, F16, } \\
\text { F17 }\end{array}$ & $\begin{array}{c}\text { F2, F3, F4, F6, F7, } \\
\text { F8, F9, F10, F11, } \\
\text { F17 }\end{array}$ & - \\
\hline F7 & F2, F3, F4, F6, F7, F8, F9, F10, F11, F17 & $\begin{array}{c}\text { F1, F2, F3, F4, F5, F6, F7, F8, F9, } \\
\text { F10, F11, F12, F13, F14, F15, F16, } \\
\text { F17 }\end{array}$ & $\begin{array}{l}\text { F2, F3, F4, F6, F7, } \\
\text { F8, F9, F10, F11, } \\
\text { F17 }\end{array}$ & - \\
\hline F8 & F2, F3, F4, F6, F7, F8, F9, F10, F11, F17 & $\begin{array}{c}\text { F1, F2, F3, F4, F5, F6, F7, F8, F9, } \\
\text { F10, F11, F12, F13, F14, F15, F16, } \\
\text { F17 }\end{array}$ & $\begin{array}{l}\mathrm{F} 2, \mathrm{~F} 3, \mathrm{~F} 4, \mathrm{~F} 6, \mathrm{~F} 7, \\
\mathrm{~F} 8, \mathrm{~F} 9, \mathrm{~F} 10, \mathrm{~F} 11, \\
\text { F17 }\end{array}$ & - \\
\hline F9 & F2, F3, F4, F6, F7, F8, F9, F10, F11, F17 & $\begin{array}{c}\text { F1, F2, F3, F4, F5, F6, F7, F8, F9, } \\
\text { F10, F11, F12, F13, F14, F15, F16, } \\
\text { F17 }\end{array}$ & $\begin{array}{l}\text { F2, F3, F4, F6, F7, } \\
\text { F8, F9, F10, F11, } \\
\text { F17 }\end{array}$ & - \\
\hline F10 & F2, F3, F4, F6, F7, F8, F9, F10, F11, F17 & $\begin{array}{c}\text { F1, F2, F3, F4, F5, F6, F7, F8, F9, } \\
\text { F10, F11, F12, F13, F14, F15, F16, } \\
\text { F17 }\end{array}$ & $\begin{array}{c}\text { F2, F3, F4, F6, F7, } \\
\text { F8, F9, F10, F11, } \\
\text { F17 }\end{array}$ & - \\
\hline F11 & F2, F3, F4, F6, F7, F8, F9, F10, F11, F17 & $\begin{array}{c}\text { F1, F2, F3, F4, F5, F6, F7, F8, F9, } \\
\text { F10, F11, F12, F13, F14, F15, F16, } \\
\text { F17 }\end{array}$ & $\begin{array}{c}\text { F2, F3, F4, F6, F7, } \\
\text { F8, F9, F10, F11, } \\
\text { F17 }\end{array}$ & - \\
\hline F12 & $\begin{array}{c}\text { F1, F2, F3, F4, F6, F7, F8, F9, F10, F11, } \\
\text { F12, F13, F14, F17 }\end{array}$ & F5, F12, F13, F14, F16 & F12, F13, F14 & - \\
\hline F13 & $\begin{array}{c}\text { F1, F2, F3, F4, F6, F7, F8, F9, F10, F11, } \\
\text { F12, F13, F14, F17 }\end{array}$ & F5, F12, F13, F14, F16 & $\mathrm{F} 12, \mathrm{~F} 13, \mathrm{~F} 14$ & - \\
\hline F14 & $\begin{array}{c}\mathrm{F} 1, \mathrm{~F} 2, \mathrm{~F} 3, \mathrm{~F} 4, \mathrm{~F} 6, \mathrm{~F} 7, \mathrm{~F} 8, \mathrm{~F} 9, \mathrm{~F} 10, \mathrm{~F} 11, \\
\mathrm{~F} 12, \mathrm{~F} 13, \mathrm{~F} 14, \mathrm{~F} 17\end{array}$ & F5, F12, F13, F14, F16 & $\mathrm{F} 12, \mathrm{~F} 13, \mathrm{~F} 14$ & - \\
\hline F15 & $\begin{array}{c}\mathrm{F} 2, \mathrm{~F} 3, \mathrm{~F} 4, \mathrm{~F} 6, \mathrm{~F} 7, \mathrm{~F} 8, \mathrm{~F} 9, \mathrm{~F} 10, \mathrm{~F} 11, \mathrm{~F} 15, \\
\text { F17 }\end{array}$ & F15 & F15 & 1 \\
\hline F16 & $\begin{array}{c}\mathrm{F} 1, \mathrm{~F} 2, \mathrm{~F} 3, \mathrm{~F} 4, \mathrm{~F} 6, \mathrm{~F} 7, \mathrm{~F} 8, \mathrm{~F} 9, \mathrm{~F} 10, \mathrm{~F} 11, \\
\mathrm{~F} 12, \mathrm{~F} 13, \mathrm{~F} 14, \mathrm{~F} 16, \mathrm{~F} 17\end{array}$ & F5, F16 & F16 & - \\
\hline F17 & F2, F3, F4, F6, F7, F8, F9, F10, F11, F17 & $\begin{array}{c}\text { F1, F2, F3, F4, F5, F6, F7, F8, F9, } \\
\text { F10, F11, F12, F13, F14, F15, F16, } \\
\text { F17 }\end{array}$ & $\begin{array}{l}\text { F2, F3, F4, F6, F7, } \\
\text { F8, F9, F10, F11, } \\
\text { F17 }\end{array}$ & - \\
\hline
\end{tabular}


The first level of the hierarchy was formed by the climatic factor and the factor of neighborhood. These factors are independent of others, but affect them. In the course of the 2nd iteration, the indicated factors (climatic factor and proximity factor) were excluded from the list of factors, and a similar procedure for determining the hierarchy levels was carried out (Table 3).

Table 3

Levels of the hierarchy of factors that affect the possibility of repurposing the urban environment and relocation of an industrial facility (2st iteration)

\begin{tabular}{|c|c|c|c|c|}
\hline Factor & $\mathrm{S}(\mathrm{zi})$ & $\mathrm{P}(\mathrm{zi})$ & $\mathrm{S}(\mathrm{zi}) \cap \mathrm{P}(\mathrm{zi})$ & $\begin{array}{l}\text { Hierarchy } \\
\text { level }\end{array}$ \\
\hline $\mathrm{F} 1$ & $\begin{array}{c}\text { F1, F2, F3, F4, F6, F7, F8, } \\
\text { F9, F10, F11, F17 }\end{array}$ & F1, F12, F13, F14, F16 & $\mathrm{F} 1$ & - \\
\hline $\mathrm{F} 2$ & $\begin{array}{c}\text { F2, F3, F4, F6, F7, F8, F9, } \\
\text { F10, F11, F17 }\end{array}$ & $\begin{array}{l}\mathrm{F} 1, \mathrm{~F} 2, \mathrm{~F} 3, \mathrm{~F} 4, \mathrm{~F} 6, \mathrm{~F} 7, \mathrm{~F} 8, \mathrm{~F} 9, \\
\mathrm{~F} 10, \mathrm{~F} 11, \mathrm{~F} 12, \mathrm{~F} 13, \mathrm{~F} 14, \mathrm{~F} 16, \\
\text { F17 }\end{array}$ & $\begin{array}{l}\text { F2, F3, F4, F6, F7, } \\
\text { F8, F9, F10, F11, } \\
\text { F17 }\end{array}$ & - \\
\hline F3 & $\begin{array}{c}\text { F2, F3, F4, F6, F7, F8, F9, } \\
\text { F10, F11, F17 }\end{array}$ & $\begin{array}{c}\mathrm{F} 1, \mathrm{~F} 2, \mathrm{~F} 3, \mathrm{~F} 4, \mathrm{~F} 6, \mathrm{~F} 7, \mathrm{~F} 8, \mathrm{~F} 9, \\
\text { F10, F11, F12, F13, F14, F16, } \\
\text { F17 }\end{array}$ & $\begin{array}{l}\text { F2, F3, F4, F6, F7, } \\
\text { F8, F9, F10, F11, } \\
\text { F17 }\end{array}$ & - \\
\hline $\mathrm{F} 4$ & $\begin{array}{c}\text { F2, F3, F4, F6, F7, F8, F9, } \\
\text { F10, F11, F17 }\end{array}$ & $\begin{array}{c}\mathrm{F} 1, \mathrm{~F} 2, \mathrm{~F} 3, \mathrm{~F} 4, \mathrm{~F} 6, \mathrm{~F} 7, \mathrm{~F} 8, \mathrm{~F} 9, \\
\text { F10, F11, F12, F13, F14, F16, } \\
\text { F17 }\end{array}$ & $\begin{array}{l}\text { F2, F3, F4, F6, F7, } \\
\text { F8, F9, F10, F11, } \\
\text { F17 }\end{array}$ & - \\
\hline F6 & $\begin{array}{c}\text { F2, F3, F4, F6, F7, F8, F9, } \\
\text { F10, F11, F17 }\end{array}$ & $\begin{array}{c}\text { F1, F2, F3, F4, F6, F7, F8, F9, } \\
\text { F10, F11, F12, F13, F14, F16, } \\
\text { F17 }\end{array}$ & $\begin{array}{l}\text { F2, F3, F4, F6, F7, } \\
\text { F8, F9, F10, F11, } \\
\text { F17 }\end{array}$ & - \\
\hline F7 & $\begin{array}{c}\text { F2, F3, F4, F6, F7, F8, F9, } \\
\text { F10, F11, F17 }\end{array}$ & $\begin{array}{c}\mathrm{F} 1, \mathrm{~F} 2, \mathrm{~F} 3, \mathrm{~F} 4, \mathrm{~F} 6, \mathrm{~F} 7, \mathrm{~F} 8, \mathrm{~F} 9, \\
\text { F10, F11, F12, F13, F14, F16, } \\
\text { F17 }\end{array}$ & $\begin{array}{l}\text { F2, F3, F4, F6, F7, } \\
\text { F8, F9, F10, F11, } \\
\text { F17 }\end{array}$ & - \\
\hline F8 & $\begin{array}{c}\text { F2, F3, F4, F6, F7, F8, F9, } \\
\text { F10, F11, F17 }\end{array}$ & $\begin{array}{c}\text { F1, F2, F3, F4, F6, F7, F8, F9, } \\
\text { F10, F11, F12, F13, F14, F17 }\end{array}$ & $\begin{array}{l}\text { F2, F3, F4, F6, F7, } \\
\text { F8, F9, F10, F11, } \\
\text { F17 }\end{array}$ & - \\
\hline F9 & $\begin{array}{c}\text { F2, F3, F4, F6, F7, F8, F9, } \\
\text { F10, F11, F17 }\end{array}$ & $\begin{array}{c}\text { F1, F2, F3, F4, F6, F7, F8, F9, } \\
\text { F10, F11, F12, F13, F14, F16, } \\
\text { F17 }\end{array}$ & $\begin{array}{l}\text { F2, F3, F4, F6, F7, } \\
\text { F8, F9, F10, F11, } \\
\text { F17 }\end{array}$ & - \\
\hline F10 & $\begin{array}{c}\text { F2, F3, F4, F6, F7, F8, F9, } \\
\text { F10, F11, F17 }\end{array}$ & $\begin{array}{c}\text { F1, F2, F3, F4, F6, F7, F8, F9, } \\
\text { F10, F11, F12, F13, F14, F16, } \\
\text { F17 }\end{array}$ & $\begin{array}{l}\text { F2, F3, F4, F6, F7, } \\
\text { F8, F9, F10, F11, } \\
\text { F17 }\end{array}$ & - \\
\hline F11 & $\begin{array}{c}\text { F2, F3, F4, F6, F7, F8, F9, } \\
\text { F10, F11, F17 }\end{array}$ & $\begin{array}{l}\text { F1, F2, F3, F4, F6, F7, F8, F9, } \\
\text { F10, F11, F12, F13, F14, F16, } \\
\text { F17 }\end{array}$ & $\begin{array}{l}\text { F2, F3, F4, F6, F7, } \\
\text { F8, F9, F10, F11, } \\
\text { F17 }\end{array}$ & - \\
\hline F12 & $\begin{array}{c}\text { F1, F2, F3, F4, F6, F7, F8, } \\
\text { F9, F10, F11, F12, F13, } \\
\text { F14, F17 }\end{array}$ & F12, F13, F14, F16 & F12, F13, F14 & - \\
\hline F13 & $\begin{array}{c}\text { F1, F2, F3, F4, F6, F7, F8, } \\
\text { F9, F10, F11, F12, F13, } \\
\text { F14, F17 }\end{array}$ & F12, F13, F14, F16 & F12, F13, F14 & - \\
\hline F14 & $\begin{array}{c}\text { F1, F2, F3, F4, F6, F7, F8, } \\
\text { F9, F10, F11, F12, F13, } \\
\text { F14, F17 }\end{array}$ & $\mathrm{F} 12, \mathrm{~F} 13, \mathrm{~F} 14, \mathrm{~F} 16$ & F12, F13, F14 & - \\
\hline F16 & $\begin{array}{c}\text { F1, F2, F3, F4, F6, F7, F8, } \\
\text { F9, F10, F11, F12, F13, } \\
\text { F14, F16, F17 }\end{array}$ & F16 & F16 & 2 \\
\hline F17 & $\begin{array}{c}\text { F2, F3, F4, F6, F7, F8, F9, } \\
\text { F10, F11, F17 }\end{array}$ & $\begin{array}{c}\text { F1, F2, F3, F4, F6, F7, F8, F9, } \\
\text { F10, F11, F12, F13, F14, F16, } \\
\text { F17 }\end{array}$ & $\begin{array}{l}\text { F2, F3, F4, F6, F7, } \\
\text { F8, F9, F10, F11, } \\
\text { F17 }\end{array}$ & - \\
\hline
\end{tabular}


The topological factor (F16) formed the second level of the hierarchy. This factor depends on the climatic and affects the implementation of factors of the availability of natural, labor resources, the development of science in the region, the climatic, factor of the investment attractiveness of the region, the presence of sales markets, competition, the development of production, engineering, transport, economic, social, tourism infrastructure, information and communication technologies, environmental friendliness of the region, the factor of the historical and cultural value of the region, the legislative factor. The definition of factors of the 3rd level of the hierarchy is presented in Table 4.

\section{Table 4}

Levels of the hierarchy of factors that affect the possibility of repurposing the urban environment and relocation of an industrial facility (3st iteration)

\begin{tabular}{|c|c|c|c|c|}
\hline Factor & $\mathrm{S}(\mathrm{zi})$ & $\mathrm{P}(\mathrm{zi})$ & $\mathrm{S}(\mathrm{zi}) \cap \mathrm{P}(\mathrm{zi})$ & $\begin{array}{c}\text { Hierarchy } \\
\text { level }\end{array}$ \\
\hline F1 & $\begin{array}{c}\text { F1, F2, F3, F4, F6, F7, F8, } \\
\text { F9, F10, F11, F17 }\end{array}$ & F1, F12, F13, F14 & $\mathrm{F} 1$ & - \\
\hline F2 & $\begin{array}{c}\text { F2, F3, F4, F6, F7, F8, F9, } \\
\text { F10, F11, F17 }\end{array}$ & $\begin{array}{c}\text { F1, F2, F3, F4, F6, F7, F8, F9, } \\
\text { F10, F11, F12, F13, F14, F17 }\end{array}$ & $\begin{array}{l}\text { F2, F3, F4, F6, F7, F8, } \\
\text { F9, F10, F11, F17 }\end{array}$ & - \\
\hline F3 & $\begin{array}{c}\text { F2, F3, F4, F6, F7, F8, F9, } \\
\text { F10, F11, F17 }\end{array}$ & $\begin{array}{c}\text { F1, F2, F3, F4, F6, F7, F8, F9, } \\
\text { F10, F11, F12, F13, F14, F17 }\end{array}$ & $\begin{array}{l}\text { F2, F3, F4, F6, F7, F8, } \\
\text { F9, F10, F11, F17 }\end{array}$ & - \\
\hline $\mathrm{F} 4$ & $\begin{array}{c}\text { F2, F3, F4, F6, F7, F8, F9, } \\
\text { F10, F11, F17 }\end{array}$ & $\begin{array}{l}\text { F1, F2, F3, F4, F6, F7, F8, F9, } \\
\text { F10, F11, F12, F13, F14, F17 }\end{array}$ & $\begin{array}{l}\text { F2, F3, F4, F6, F7, F8, } \\
\text { F9, F10, F11, F17 }\end{array}$ & - \\
\hline F6 & $\begin{array}{c}\text { F2, F3, F4, F6, F7, F8, F9, } \\
\text { F10, F11, F17 }\end{array}$ & $\begin{array}{l}\text { F1, F2, F3, F4, F6, F7, F8, F9, } \\
\text { F10, F11, F12, F13, F14, F17 }\end{array}$ & $\begin{array}{l}\text { F2, F3, F4, F6, F7, F8, } \\
\text { F9, F10, F11, F17 }\end{array}$ & - \\
\hline F7 & $\begin{array}{c}\text { F2, F3, F4, F6, F7, F8, F9, } \\
\text { F10, F11, F17 }\end{array}$ & $\begin{array}{l}\text { F1, F2, F3, F4, F6, F7, F8, F9, } \\
\text { F10, F11, F12, F13, F14, F17 }\end{array}$ & $\begin{array}{l}\text { F2, F3, F4, F6, F7, F8, } \\
\text { F9, F10, F11, F17 }\end{array}$ & - \\
\hline F8 & $\begin{array}{c}\text { F2, F3, F4, F6, F7, F8, F9, } \\
\text { F10, F11, F17 }\end{array}$ & $\begin{array}{l}\text { F1, F2, F3, F4, F6, F7, F8, F9, } \\
\text { F10, F11, F12, F13, F14, F17 }\end{array}$ & $\begin{array}{l}\text { F2, F3, F4, F6, F7, F8, } \\
\text { F9, F10, F11, F17 }\end{array}$ & - \\
\hline F9 & $\begin{array}{c}\text { F2, F3, F4, F6, F7, F8, F9, } \\
\text { F10, F11, F17 }\end{array}$ & $\begin{array}{l}\text { F1, F2, F3, F4, F6, F7, F8, F9, } \\
\text { F10, F11, F12, F13, F14, F17 }\end{array}$ & $\begin{array}{l}\text { F2, F3, F4, F6, F7, F8, } \\
\text { F9, F10, F11, F17 }\end{array}$ & - \\
\hline F10 & $\begin{array}{c}\text { F2, F3, F4, F6, F7, F8, F9, } \\
\text { F10, F11, F17 }\end{array}$ & $\begin{array}{l}\text { F1, F2, F3, F4, F6, F7, F8, F9, } \\
\text { F10, F11, F12, F13, F14, F17 }\end{array}$ & $\begin{array}{l}\text { F2, F3, F4, F6, F7, F8, } \\
\text { F9, F10, F11, F17 }\end{array}$ & - \\
\hline F11 & $\begin{array}{c}\text { F2, F3, F4, F6, F7, F8, F9, } \\
\text { F10, F11, F17 }\end{array}$ & $\begin{array}{l}\text { F1, F2, F3, F4, F6, F7, F8, F9, } \\
\text { F10, F11, F12, F13, F14, F17 }\end{array}$ & $\begin{array}{l}\text { F2, F3, F4, F6, F7, F8, } \\
\text { F9, F10, F11, F17 }\end{array}$ & - \\
\hline F12 & $\begin{array}{c}\text { F1, F2, F3, F4, F6, F7, F8, } \\
\text { F9, F10, F11, F12, F13, } \\
\text { F14, F17 }\end{array}$ & F12, F13, F14 & F12, F13, F14 & 3 \\
\hline F13 & $\begin{array}{c}\text { F1, F2, F3, F4, F6, F7, F8, } \\
\text { F9, F10, F11, F12, F13, } \\
\text { F14, F17 }\end{array}$ & F12, F13, F14 & F12, F13, F14 & 3 \\
\hline F14 & $\begin{array}{c}\text { F1, F2, F3, F4, F6, F7, F8, } \\
\text { F9, F10, F11, F12, F13, } \\
\text { F14, F17 }\end{array}$ & F12, F13, F14 & F12, F13, F14 & 3 \\
\hline F17 & $\begin{array}{c}\text { F2, F3, F4, F6, F7, F8, F9, } \\
\text { F10, F11, F17 }\end{array}$ & $\begin{array}{l}\text { F1, F2, F3, F4, F6, F7, F8, F9, } \\
\text { F10, F11, F12, F13, F14, F17 }\end{array}$ & $\begin{array}{c}\text { F2, F3, F4, F6, F7, F8, } \\
\text { F9, F10, F11, F17 }\end{array}$ & - \\
\hline
\end{tabular}

At the 3rd level of the hierarchy, the factors of environmental friendliness of the region, the historical and cultural value of the region, the legislative factor are highlighted. Table 5 shows the factors of the 4 th level of the hierarchy. 
Table 5.

Levels of the hierarchy of factors that affect the possibility of repurposing the urban environment and relocation of an industrial facility (4st iteration)

\begin{tabular}{|c|c|c|c|c|}
\hline Factor & $\mathrm{S}(\mathrm{zi})$ & $\mathrm{P}(\mathrm{zi})$ & $\mathrm{S}(\mathrm{zi}) \cap \mathrm{P}(\mathrm{zi})$ & $\begin{array}{c}\text { Hierarchy } \\
\text { level }\end{array}$ \\
\hline $\mathrm{F} 1$ & $\begin{array}{c}\text { F1, F2, F3, F4, F6, F7, F8, } \\
\text { F9, F10, F11, F17 }\end{array}$ & $\mathrm{F} 1$ & $\mathrm{~F} 1$ & 4 \\
\hline $\mathrm{F} 2$ & $\begin{array}{c}\text { F2, F3, F4, F6, F7, F8, F9, } \\
\text { F10, F11, F17 }\end{array}$ & $\begin{array}{c}\text { F1, F2, F3, F4, F6, F7, F8, } \\
\text { F9, F10, F11, F17 }\end{array}$ & $\begin{array}{c}\text { F2, F3, F4, F6, F7, F8, } \\
\text { F9, F10, F11, F17 }\end{array}$ & - \\
\hline $\mathrm{F} 3$ & $\begin{array}{c}\text { F2, F3, F4, F6, F7, F8, F9, } \\
\text { F10, F11, F17 }\end{array}$ & $\begin{array}{c}\text { F1, F2, F3, F4, F6, F7, F8, } \\
\text { F9, F10, F11, F17 }\end{array}$ & $\begin{array}{l}\text { F2, F3, F4, F6, F7, F8, } \\
\text { F9, F10, F11, F17 }\end{array}$ & - \\
\hline $\mathrm{F} 4$ & $\begin{array}{c}\text { F2, F3, F4, F6, F7, F8, F9, } \\
\text { F10, F11, F17 }\end{array}$ & $\begin{array}{c}\mathrm{F} 1, \mathrm{~F} 2, \mathrm{~F} 3, \mathrm{~F} 4, \mathrm{~F} 6, \mathrm{~F} 7, \mathrm{~F} 8, \\
\mathrm{~F} 9, \mathrm{~F} 10, \mathrm{~F} 11, \mathrm{~F} 17\end{array}$ & $\begin{array}{c}\text { F2, F3, F4, F6, F7, F8, } \\
\text { F9, F10, F11, F17 }\end{array}$ & - \\
\hline F6 & $\begin{array}{c}\text { F2, F3, F4, F6, F7, F8, F9, } \\
\text { F10, F11, F17 }\end{array}$ & $\begin{array}{c}\text { F1, F2, F3, F4, F6, F7, F8, } \\
\text { F9, F10, F11, F17 }\end{array}$ & $\begin{array}{l}\text { F2, F3, F4, F6, F7, F8, } \\
\text { F9, F10, F11, F17 }\end{array}$ & - \\
\hline F7 & $\begin{array}{c}\text { F2, F3, F4, F6, F7, F8, F9, } \\
\text { F10, F11, F17 }\end{array}$ & $\begin{array}{c}\text { F1, F2, F3, F4, F6, F7, F8, } \\
\text { F9, F10, F11, F17 }\end{array}$ & $\begin{array}{l}\text { F2, F3, F4, F6, F7, F8, } \\
\text { F9, F10, F11, F17 }\end{array}$ & - \\
\hline F8 & $\begin{array}{c}\text { F2, F3, F4, F6, F7, F8, F9, } \\
\text { F10, F11, F17 }\end{array}$ & $\begin{array}{c}\text { F1, F2, F3, F4, F6, F7, F8, } \\
\text { F9, F10, F11, F17 }\end{array}$ & $\begin{array}{l}\text { F2, F3, F4, F6, F7, F8, } \\
\text { F9, F10, F11, F17 }\end{array}$ & - \\
\hline F9 & $\begin{array}{c}\text { F2, F3, F4, F6, F7, F8, F9, } \\
\text { F10, F11, F17 }\end{array}$ & $\begin{array}{c}\text { F1, F2, F3, F4, F6, F7, F8, } \\
\text { F9, F10, F11, F17 }\end{array}$ & $\begin{array}{l}\text { F2, F3, F4, F6, F7, F8, } \\
\text { F9, F10, F11, F17 }\end{array}$ & - \\
\hline F10 & $\begin{array}{c}\text { F2, F3, F4, F6, F7, F8, F9, } \\
\text { F10, F11, F17 }\end{array}$ & $\begin{array}{c}\mathrm{F} 1, \mathrm{~F} 2, \mathrm{~F} 3, \mathrm{~F} 4, \mathrm{~F} 6, \mathrm{~F} 7, \mathrm{~F} 8, \\
\mathrm{~F} 9, \mathrm{~F} 10, \mathrm{~F} 11, \mathrm{~F} 17\end{array}$ & $\begin{array}{c}\text { F2, F3, F4, F6, F7, F8, } \\
\text { F9, F10, F11, F17 }\end{array}$ & - \\
\hline F11 & $\begin{array}{c}\text { F2, F3, F4, F6, F7, F8, F9, } \\
\text { F10, F11, F17 }\end{array}$ & $\begin{array}{c}\text { F1, F2, F3, F4, F6, F7, F8, } \\
\text { F9, F10, F11, F17 }\end{array}$ & $\begin{array}{l}\text { F2, F3, F4, F6, F7, F8, } \\
\text { F9, F10, F11, F17 }\end{array}$ & - \\
\hline F17 & $\begin{array}{c}\text { F2, F3, F4, F6, F7, F8, F9, } \\
\text { F10, F11, F17 }\end{array}$ & $\begin{array}{c}\mathrm{F} 1, \mathrm{~F} 2, \mathrm{~F} 3, \mathrm{~F} 4, \mathrm{~F} 6, \mathrm{~F} 7, \mathrm{~F} 8, \\
\mathrm{~F} 9, \mathrm{~F} 10, \mathrm{~F} 11, \mathrm{~F} 17\end{array}$ & $\begin{array}{c}\mathrm{F} 2, \mathrm{~F} 3, \mathrm{~F} 4, \mathrm{~F} 6, \mathrm{~F} 7, \mathrm{~F} 8, \\
\mathrm{~F} 9, \mathrm{~F} 10, \mathrm{~F} 11, \mathrm{~F} 17\end{array}$ & - \\
\hline
\end{tabular}

At the 4th level of the hierarchy, the factor of the availability of natural resources is highlighted. This factor affects the investment attractiveness of the region, the development of production, engineering, transport, economic, social, and tourist infrastructure. Table 6 shows the factors affecting the possibility of re-profiling the urban environment and relocation of an industrial facility, which formed the 5th level of the hierarchy.

Table 6.

Levels of the hierarchy of factors that affect the possibility of repurposing the urban environment and relocation of an industrial facility (5st iteration)

\begin{tabular}{|c|c|c|c|c|}
\hline Factor & $\mathrm{S}(\mathrm{zi})$ & $\mathrm{P}(\mathrm{zi})$ & $\mathrm{S}(\mathrm{zi}) \cap \mathrm{P}(\mathrm{zi})$ & $\begin{array}{c}\text { Hierarchy } \\
\text { level }\end{array}$ \\
\hline $\mathrm{F} 2$ & $\begin{array}{c}\text { F2, F3, F4, F6, F7, F8, F9, } \\
\text { F10, F11, F17 }\end{array}$ & $\begin{array}{c}\mathrm{F} 2, \mathrm{~F} 3, \mathrm{~F} 4, \mathrm{~F} 6, \mathrm{~F} 7, \mathrm{~F} 8, \mathrm{~F} 9, \\
\text { F10, F11, F17 }\end{array}$ & $\begin{array}{c}\text { F2, F3, F4, F6, F7, F8, } \\
\text { F9, F10, F11, F17 }\end{array}$ & 5 \\
\hline F3 & $\begin{array}{c}\text { F2, F3, F4, F6, F7, F8, F9, } \\
\text { F10, F11, F17 }\end{array}$ & $\begin{array}{c}\mathrm{F} 2, \mathrm{~F} 3, \mathrm{~F} 4, \mathrm{~F} 6, \mathrm{~F} 7, \mathrm{~F} 8, \mathrm{~F} 9, \\
\mathrm{~F} 10, \mathrm{~F} 11, \mathrm{~F} 17\end{array}$ & $\begin{array}{c}\text { F2, F3, F4, F6, F7, F8, } \\
\text { F9, F10, F11, F17 }\end{array}$ & 5 \\
\hline $\mathrm{F} 4$ & $\begin{array}{c}\text { F2, F3, F4, F6, F7, F8, F9, } \\
\text { F10, F11, F17 }\end{array}$ & $\begin{array}{c}\text { F2, F3, F4, F6, F7, F8, F9, } \\
\text { F10, F11, F17 }\end{array}$ & $\begin{array}{l}\text { F2, F3, F4, F6, F7, F8, } \\
\text { F9, F10, F11, F17 }\end{array}$ & 5 \\
\hline F6 & $\begin{array}{c}\text { F2, F3, F4, F6, F7, F8, F9, } \\
\text { F10, F11, F17 }\end{array}$ & $\begin{array}{c}\mathrm{F} 2, \mathrm{~F} 3, \mathrm{~F} 4, \mathrm{~F} 6, \mathrm{~F} 7, \mathrm{~F} 8, \mathrm{~F} 9, \\
\mathrm{~F} 10, \mathrm{~F} 11, \mathrm{~F} 17\end{array}$ & $\begin{array}{c}\text { F2, F3, F4, F6, F7, F8, } \\
\text { F9, F10, F11, F17 }\end{array}$ & 5 \\
\hline F7 & $\begin{array}{c}\text { F2, F3, F4, F6, F7, F8, F9, } \\
\text { F10, F11, F17 }\end{array}$ & $\begin{array}{c}\text { F2, F3, F4, F6, F7, F8, F9, } \\
\text { F10, F11, F17 }\end{array}$ & $\begin{array}{l}\text { F2, F3, F4, F6, F7, F8, } \\
\text { F9, F10, F11, F17 }\end{array}$ & 5 \\
\hline F8 & $\begin{array}{c}\text { F2, F3, F4, F6, F7, F8, F9, } \\
\text { F10, F11, F17 }\end{array}$ & $\begin{array}{c}\mathrm{F} 2, \mathrm{~F} 3, \mathrm{~F} 4, \mathrm{~F} 6, \mathrm{~F} 7, \mathrm{~F} 8, \mathrm{~F} 9, \\
\mathrm{~F} 10, \mathrm{~F} 11, \mathrm{~F} 17\end{array}$ & $\begin{array}{c}\text { F2, F3, F4, F6, F7, F8, } \\
\text { F9, F10, F11, F17 }\end{array}$ & 5 \\
\hline F9 & $\begin{array}{c}\text { F2, F3, F4, F6, F7, F8, F9, } \\
\text { F10, F11, F17 }\end{array}$ & $\begin{array}{c}\text { F2, F3, F4, F6, F7, F8, F9, } \\
\text { F10, F11, F17 }\end{array}$ & $\begin{array}{c}\text { F2, F3, F4, F6, F7, F8, } \\
\text { F9, F10, F11, F17 }\end{array}$ & 5 \\
\hline
\end{tabular}




\begin{tabular}{ccccc} 
F10 & F2, F3, F4, F6, F7, F8, F9, & F2, F3, F4, F6, F7, F8, F9, & F2, F3, F4, F6, F7, F8, & 5 \\
& F10, F11, F17 & F10, F11, F17 & F9, F10, F11, F17 & \\
F11 & F2, F3, F4, F6, F7, F8, F9, & F2, F3, F4, F6, F7, F8, F9, & F2, F3, F4, F6, F7, F8, & 5 \\
F10, F11, F17 & F10, F11, F17 & F9, F10, F11, F17 & \\
F17 & F2, F3, F4, F6, F7, F8, F9, & F2, F3, F4, F6, F7, F8, F9, & F2, F3, F4, F6, F7, F8, & 5 \\
\hline
\end{tabular}

Based on the results of the 5th iteration, the factors that are the resultant in assessing the possibility of re-profiling the urban environment and relocation of an industrial facility were identified. This is the factor in the availability of labor resources and their cost; the factor of access to technology; the factor in the development of science in the region; the factor of investment attractiveness of the region; the factor of the availability of sales markets; the factor of competition; the factor in the development of production, engineering, transport, economic infrastructure; the factor in the development of social, tourist infrastructure; the factor in the development of information and communication technologies; and the personal factor.

\section{Discussion}

Thus, within the framework of the conducted research, an econometric hierarchical model was developed for the classification of factors affecting the possibility of re-profiling the urban environment and relocation of an industrial facility, which is a tool for tactical planning and monitoring of the activities of industrial facilities, as well as a tool for planning the development of a region. The proposed model, in contrast to the existing results of earlier studies (Lai et al., 2020; Wang, 2019; Ge, Qin, Li, 2018), is universal when deciding on the possibility of repurposing the urban environment and relocating an industrial facility in Russia to connection with the universal nature of the evaluated factors. Applicable to other countries, it is possible to adjust the model taking into account the national legislation of other countries and adapt the model to specific business conditions by changing the list of the most significant factors.

The assessment made it possible to identify a 5-level structure of factors that affect the possibility of re-profiling the urban environment and relocation of an industrial facility. The highest level (5th level) of factors that reflect the influence of other factors and thus are the resultant when deciding on the possibility of re-profiling the urban environment and relocation of an industrial facility, is represented by such factors: the factor of the availability of labor resources and their cost; the factor of access to technology; the factor in the development of science in the region; the factor of investment attractiveness of the region; the factor of the availability of sales markets; the factor of competition; the factor in the development of production, engineering, transport, economic infrastructure; the factor in the development of 
social, tourist infrastructure; the factor in the development of information and communication technologies; the personal factor. The hierarchical model utilizes the lack of fragmentariness of previous studies (Lai et al., 2020; Wang, 2019; Ge, Qin, Li, 2018) in determining the factors of repurposing the urban environment and redevelopment of industrial facilities, as it is based on an integrated approach and allows to take into account prioritizing a wide range of underlying factors.

It has been found that the organization of territorial and spatial distribution of the main functional processes of the modern post-industrial settlement system (cities and agglomerations) is multifactorial and complex in most cases. The modern urban structure (as a special form of displaying the material and spatial environment) functions in the format of an integral social and industrial complex and constitutes (together with engineering, transport, landscape, and environmental frameworks) a single object of the urban design.

The most frequent phenomenon is the presence of such a town-planning situation that is formed in some local part of the urban system conditions to ensure the state and/or sustainable development of one dominant function (the corresponding territorial zone is characterized as monofunctional) (Surya et al., 2019).

Moreover, it has been found that composition and hierarchy of the subordination of the structural elements forming the industrial environment depended on the specific climatic, urban planning, and large-scale technological features of industrial production and functional relationships with the space of the urban environment. Therefore, industrial sites and zones included in the urban structure could be considered as centers of gravity and urban elements of a special composite value (composite centers and dominant) have been interconnected with other functional areas (primarily residential environment) of the relevant spatial and communications links (open spaces, road network, and utility networks).

\section{Conclusion}

The effective organization of the use of the territory of an urban environment, which is suitable and accessible for building construction facilities for industrial purposes is an urgent task for existing, new, and reconstructed industrial zones. The use of the urban environment to place the production facilities has been directly related to the characteristics of a particular type of industrial industry, the scale of the need for production activities, and the permissible level of negative impact on the environment. However, requirements of developing some branches of management (real economy of the industrial period) led to the necessity of formation of such systems of resettlement, in which the structure and functioning of industrial zones have acquired the importance of the main city-forming factor, to which the structure and functionally- 
territorial balance of the territory available for the building have been put in hierarchical subordination.

In addition, a significant share of industrial zones in the balance of functional saturation of the territory of the urban structure clearly indicated an obligation to include the industrial zone in the composition and architectural planning decisions (on the organization of space and connections with other functional zones) of a single and integral urban environment. This circumstance has been equally relevant for reorganization and development of the existing settlement system, as well as the development of new and accessible areas for urban development.

\section{References}

Abid, N.M., Saeed, A.A., Al-Beyaty, S.F.G. (2019). Trends in the Physical and Social Urban Form: Policy-Making and Natural Sustainable Development Dialectics. Journal of Southwest Jiaotong University, 54(6). http://jsju.org/index.php/journal/article/view/460

Abramov, I. (2018). Formation of integrated structural units using the systematic and integrated method when implementing high-rise construction projects. HRC 2017 (HIGH-RISE CONSTRUCTION-2017) E3S Web of Conferences, 2018, 33, 1-7.

Aleskerov, Z. (2019). What you shouldn't forget when redeveloping industrial zones in Moscow. RBC. Retrieved from: https://realty.rbc.ru/news/5dfa25649a7947168cd0fe3d

Auzan, A. (2017). Revolutions and evolutions in Russia: In search of a solution to the path dependence problem. Russian Journal of Economics, 3(4), 336-347. https://doi.org/10.1016/j.ruje.2017.12.002.

Bieri, D. (2019). Hamlet without the Prince? August Lösch and how Spatial Economics Abandoned Monetary Analysis. European Society for the History of Economic Thought (Annual Meetings). At: Lille, France. Retrieved from: https://www.researchgate.net/publication/337155540_Hamlet_without the Prince_Au gust_Losch_and_how_Spatial_Economics_Abandoned_Monetary_Analysis

Braila, N., Iatsinevich, P., Korenevskaya, M., Erzakov, S., Simankina, T. (2018). Reconstruction of industrial building with nonstandard space planning decisions. IOP Conf. Series: Materials Science and Engineering, 365, 022045. https://doi.org/10.1088/1757-899X/365/2/022045.

Cristóbal, J.R., Carral, L., Diaz, E., Fraguela, J.A., Iglesias, G. (2018). Complexity and Project Management: Challenges, Opportunities, and Future Research. Complexity, 4891286. https://doi.org/10.1155/2018/4891286.

Deloitte CIS Research Center. (2018). Overview of Manufacturing Industry in Russia. Industry 4.0: are the manufacturing companies ready? Retrieved from: https://www2.deloitte.com/ru/en/pages/manufacturing/articles/overview-ofmanufacturing-industry-in-Russia.html 
Ge, L., Qin, B., Li, S. (2018) Industrial layout reconstruction, the role of local government and variation of pollution distribution in China. Chinese Journal of Population Resources and Environment, 16(4), 314-328. https://doi.org/10.1080/10042857.2018.1544751

Gokhberg, L., Kuzminov, I., Khabirova, E., Thurner, T. (2020). Advanced text-mining for trend analysis of Russia's Extractive Industries. Futures, 115, 102476. https://doi.org/10.1016/j.futures.2019.102476.

Gubina, O.V., Provorova, A.A. (2019). Approaches and Principles for Scenarios of Spatial Ecological and Economic Development of the Russian Arctic Regions. Modern problems of science and education, 11(1), 39-47. Retrieved from: https://vaael.ru/en/article/view?id=787

Ilyichev V.A., Nikiforova N.S., Konnov A.V. (2017). A settlement calculation for neighbouring buildings with mitigation measures upon underground construction. Proceedings of 19th International Conference on Soil, Mechanics and Geotechnical Engineering, 1789-1792.

Julianelli, V., Caiado, R., Scavarda, L.F., Cruz, S. (2020). The interplay between reverse logistics and circular economy: Critical success factors-based taxonomy and framework. Resources, Conservation and Recycling, 158, 104784. https://doi.org/10.1016/j.resconrec.2020.104784.

Kearney, A.T. (2017). Technology and Innovation for the Future of Production: Accelerating Value Creation. World Economic Forum. Retrieved from: http://www3.weforum.org/docs/WEF_White_Paper_Technology_Innovation_Future of_Production_2017.pdf

Kosyakov, D., Guskov, A. (2019). Research assessment and evaluation in Russian fundamental science. Procedia Computer Science, 146, 11-19. https://doi.org/10.1016/j.procs.2019.01.072.

Kühn, D., Osthus, D., Townsend, T., \& Zhao, Yi. (2017). On the structure of oriented graphs and digraphs with forbidden tournaments or cycles. Journal of Combinatorial Theory, $B(124), 88-127$

Lai, Y., Chen, K., Zhang, J., Liu, F. (2020). Transformation of Industrial Land in Urban Renewal in Shenzhen, China. Land, 9, 371. https://doi.org/10.3390/land9100371.

Lapidus, A., Abramov, I. (2018). Formation of production structural units within a construction company using the systemic integrated method when implementing high-rise development projects. E3S Web of Conferences, 33.

Lapidus, A., Topchiy, D. (2019). Formation of Methods for Assessing the Effectiveness of Industrial Areas' Renovation Projects. Proceedings of the IOP Conference Series: Materials Science and Engineering, 471, 1-6.

Lapidus, A.A. (2020). Creating a structure to calculate the organizational and technological process control effectiveness during redeployment. Revista Ingeniería, 27, 1, 84-90. Retrieved from: https://www.redalyc.org/jatsRepo/707/70763088010/html/index.html 
Lonati, G., Saponaro, S., Sezenna, E. (2019). Vapor intrusion evaluation for the redevelopment of former industrial facilities. IOP Conf. Ser.: Earth Environ. Sci., 296, 012011. Retrieved from: https://iopscience.iop.org/article/10.1088/1755$\underline{1315 / 296 / 1 / 012011 .}$

Magnusson, T., Berggren, C. (2018). Competing innovation systems and the need for redeployment in sustainability transitions. Technological Forecasting and Social Change, 126, 217-230. https://doi.org/10.1016/j.techfore.2017.08.014.

Mäkitie, T. (2019). Sustainability transitions in oil economies: Resource redeployment from an established industry to a clean technology industry. https://doi.org/10.13140/RG.2.2.11601.97128.

Nagy, J., Oláh, J., Erdei, E., Máté, D., Popp, J. (2018). The Role and Impact of Industry 4.0 and the Internet of Things on the Business Strategy of the Value Chain - The Case of Hungary. Sustainability, 10, 3491. https://doi.org/10.3390/su10103491.

Pajula, T., Behm, K., Vatanen, S., Saarivuori, E. (2017). Managing the Life Cycle to Reduce Environmental Impacts. In: Grösser S., Reyes-Lecuona A., Granholm G. (eds) Dynamics of Long-Life Assets. Springer, Cham. https://doi.org/10.1007/978-3-319$\underline{45438-2 \_6}$

Pezeshki, Z., Ivari, S.A.S. (2018). Applications of BIM: A Brief Review and Future Outline. Archives of Computational Methods in Engineering, 25(2), 273-312.

Power, A. (2018). Regional politics of an urban age: Can Europe's former industrial cities create a new industrial economy to combat climate change and social unravelling? Palgrave Commun, 4, 97. https://doi.org/10.1057/s41599-018-0120-x

Reiffenstein, T. (2017). Concentric Zone Theory. https://doi.org/10.1002/9781118430873.est0440

Surya, B., Saleh, H., Syafri, Ahmad, D.N.A. (2019). Impact and Sustainability of New Urban Area Development in Moncongloe-Pattalassang, Mamminasata Metropolitan. Journal $\begin{array}{llll}\text { of Southwest Jiaotong } & \text { University, }\end{array}$ http://jsju.org/index.php/journal/article/view/462

Telichenko, V. I., Gnyrya, A. I., Boyarintsev, A. P. (2018). Construction technology for highrise, large-span, special buildings and structures. Moscow: ASV publishing house.

Topchiy, D., Yurgaitis, A., Kravchuk, A., Shevchuk, D. (2019). Controlling methods of buildings' energy performance characteristics. Topical Problems of Architecture, Civil Engineering and Environmental Economics (TPACEE 2018) electronic edition. E3S Web of Conferences.

Wang, T. (2019). A sustainable industrial site redevelopment planning support system. TechnischeUniversiteit Eindhoven. Retrieved from: https://pure.tue.nl/ws/files/119666561/20190402_Wang.pdf

Yanitsky, O. (2018). Russian Megalopolis: Processes of Divergence, Separation and Integration of its Agents. International Journal of Political Science, 4(3), 5-13. http://dx.doi.org/10.20431/2454-9452.0403002. 